Hence the commendable tendency to amputate at the higher level when in doubt. Similarly a failure to walk with a prosthesis is a failure by the rehabilitationist, and much can be gained for the patient by closer co-operation between all those responsible for his care.

\section{Otitis Externa}

Infection of the skin lining the external auditory canal occurs most often in hot and humid atmospheric conditions, and bathing in a hot climate with high humidity is a potent cause. The chronic form may result from inadequate treatment of the acute disease, but in Britain other factors are more usual. Seborrhoea, eczema, or mild emotional stress are found in a high proportion of cases. The general use of preparations containing broad spectrum antibiotics and anti-inflammatory steroids has led to a fall in the number of patients referred for specialist treatment, but the condition is still common. Some cases resistant to treatment are due to local drug sensitivity-the reaction is damped down by the steroid although discharge and slight discomfort persist.

As was pointed out by J. W. McLaurin ${ }^{1}$ and G. B. E. Beaney and A. Broughton, ${ }^{2}$ the small meatal outlet produces a skin-lined culture tube with optimum conditions for the growth of organisms. Healthy wax has an acid reaction with antiseptic properties, but if the meatus becomes alkaline the defensive mechanism is upset. The continued removal of the lipid layer by sweat or exudate from the skin layer or by the entrance of water almost certainly explains why tropical climates and bathing encourage otitis externa. The organisms causing infection cannot be eliminated if they are resistant to the antiseptic applied, if swelling of the meatal walls or debris lying in the meatus prevents the antiseptic reaching the organisms, if the epithelium is repeatedly abraded by cleansing or the insertion of dressings, or if long-continued use of an antiseptic allows the growth of other organisms resistant to its action. After a severe infection there may be sub-epithelial fibrosis of the meatal skin, which reduces the resistance to bacterial attack at a later date. However, if reinfection can be prevented this fibrosis will in time be absorbed. Certainly relapses of infection after severe tropical otitis diminish if the subject resides in a temperate climate.

A wide variety of organisms are found on culture of swabs from the ear. The commonest are Pseudomonas pyocyanea, Escherichia coli, Proteus spp., and staphylococci. A pure growth is found only in one-half or three-quarters of the cases, and in many two or more varieties are found. Candida, aspergillus, and other fungi are found in about $80 \%$ of cases of tropical otitis externa, but in Britain the proportion is smaller, perhaps $20 \%$. Before the introduction of treatment with broad spectrum antibiotics fungal infections in this country were rare in both acute and chronic cases.

When acute otitis externa has developed the severe case presents the urgent problem of relief of pain caused by the meatal oedema. Systemic antibiotic therapy will be useless if the organisms are resistant or if the infection is fungal, so

1 MaLaurin, J. W., Raggio, T. P., and Simmons, M., Laryngoscope, 1965, 75, 1699.

- Beaney, G. P. E., and Broughton, A., F. Laryng., 1967, 81, 987.

Deaney, G. P. E., and Broughton, A., f. Laryng., 1967, $81,987$. 82, 379 . local treatment is often best. Wicks of $\frac{1}{2}$ inch $(1.3 \mathrm{~cm}$.) wide selvaged gauze impregnated with an antiseptic or astringent preparation can usually be inserted without abrading the meatal walls. A wide range of substances has been recommended. Beaney and Broughton ${ }^{2}$ obtained a high proportion of cures in Aden with wicks impregnated with hydrargaphen followed after a week by the instillation of hydrargaphen tincture $0.1 \%$. In Britain fungal infection is less common, so local treatment with a combination of a broad-spectrum antibiotic and a steroid will usually succeed.

Chronic otitis externa may arise per se but may follow and conceal an underlying otitis media. It may also arise in a radical mastoid cavity or following a fenestration operation. As with acute otitis, the common infecting organisms are unlikely to respond to systemic antibiotic therapy. By the time a swab of the pus has shown the types and antibiotic sensitivities of the organisms (and 10\% of the swabs will not produce any growth on incubation) suitable local treatment will in most cases have effected considerable improvement. In either case removal of macerated epithelial debris and pus is essential for a sustained cure. The skin of the meatus readily becomes sensitized to substances applied to it. Some, such as chloramphenicol and penicillin, produce sensitivity within a few weeks in a high proportion of cases and should therefore never be used. Others, such as neomycin, are much less active and usually require application for a longer period. However, it should be accepted that any preparation except the most inert may at times result in sensitization, which may persist for long periods. It is sound practice not to use the same preparation continuously for more than two or three weeks, and if treatment is still required to ring the changes from one drug to another at short intervals. Perhaps because this has not been done in the past there is a tendency for the broad spectrum antibiotics not to be prescribed in case the patient has already become sensitized to them. Preparations with antifungal as well as antibacterial action, such as iodochlorhydroxyquinoline and halquinol, have produced good results in recent clinical trials. ${ }^{3}$ Many other preparations have been recommended for this condition, but their success is probably due at least in part to the care and thoroughness with which the canal is kept clear of debris.

Once the infection has been overcome the meatal walls should remain dry, but scales may continue to form and cause irritation. This can usually be controlled by application of a greasy based steroid ointment without any antiseptic, but failure to treat seborrhoea, eczema, local psoriasis, or an underlying emotional tension will lead to relapse and possible reinfection. The return of a coating of cerumen of normal colour and consistency generally means that the condition has subsided and no further observation is needed. For several months, or possibly for one or two years afterwards in the severe or resistant case, the patient should avoid allowing water to enter the ear while washing and be warned of the ri ks of returning to humid tropical conditions.

Members of the British Medical Association will have noted with special pleasure the knighthoods conferred in the New Year Honours on their President, Dr. Clarence Rieger, and their President-elect, Dr. J. W. Howie. A full list appears at page 111. 